Grai $n$ - si ze dependence of the rel at i onshi $p$ bet ween i nt er gr anul ar and i nt $r$ agr anul ar def or mat i on of nanocrystal I i ne Al by mol ecul ar dynami cs si mul at i ons

\begin{tabular}{|l|l|}
\hline 著者 & Shi mokawa Tonot sugu, Nakat ani A. , K t agawa H. \\
\hline $\begin{array}{l}\text { j our nal or } \\
\text { publ i cat i on ti tl e }\end{array}$ & $\begin{array}{l}\text { Physi cal Revi ew B - Condensed Nat ter and } \\
\text { Nat er i al s Physi cs }\end{array}$ \\
\hline vol une & 71 \\
\hline nunber & 22 \\
\hline page r ange & 224110-1-224110- 8 \\
\hline year & 2005- 06-01 \\
\hline URL & ht t p: //hdl . handl e. net /2297/3511 \\
\hline
\end{tabular}




\title{
Grain-size dependence of the relationship between intergranular and intragranular deformation of nanocrystalline Al by molecular dynamics simulations
}

\author{
T. Shimokawa, ${ }^{1,2}$ A. Nakatani, ${ }^{2}$ and H. Kitagawa ${ }^{2,3}$ \\ ${ }^{1}$ Division of Mechanical Science and Engineering, Graduate School of Natural Science and Technology, Kanazawa University, \\ 2-40-20 Kodatsuno, Kanazawa, Ishikawa 920-8667, Japan \\ ${ }^{2}$ Department of Adaptive Machine Systems, Graduate School of Engineering, Osaka University, 2-1 Yamadaoka, Suita, \\ Osaka 565-0871, Japan \\ ${ }^{3}$ Department of Mechanical Engineering, Faculty of Engineering, Doshisha University, Tatara Tsudani, Kyotanabe, \\ Kyoto 602-8580, Japan
}

(Received 12 August 2004; revised manuscript received 5 April 2005; published 24 June 2005)

\begin{abstract}
The strength of nanocrystalline aluminum has been studied using molecular dynamics simulation. Nanocrystalline models consisting of hexagonal grains with grain size $d$ between $5 \mathrm{~nm}$ and $80 \mathrm{~nm}$ are deformed by the application of tension. A transition from grain-size hardening to grain-size softening can be observed in the region where $d \approx 30 \mathrm{~nm}$, which is the optimum grain size for strength. In the grain-size hardening region, nanocrystalline models primarily deform by intragranular deformation. Consequently, a pile-up of dislocations can be observed. When the grain size becomes less than $30 \mathrm{~nm}$, where the thickness of the grain boundaries cannot be neglected in comparison to the grain sizes, the dominant deformation mechanism of nanocrystalline metals is intergranular deformation by grain boundary sliding. Further, geometrical misfits by grain boundary sliding are accommodated by the grain rotation mechanism. Moreover, cooperative grain boundary sliding occurs in the $5 \mathrm{~nm}$ model. The optimum grain size is controlled by the relationship between resistance to intergranular deformation by grain boundary processes and intragranular deformation resisted by the grain boundary. Therefore, the primary role of the grain boundary changes in the region where the optimum grain size is observed.
\end{abstract}

DOI: 10.1103/PhysRevB.71.224110

PACS number(s): 62.25.+g, 31.15.Qg, 62.20.Fe

\section{INTRODUCTION}

The deformation of polycrystalline metallic materials involves both intragranular and intergranular deformation. Intragranular deformation is caused primarily by the movement of dislocations, whereas intergranular deformation is caused by grain boundary sliding, migration, and diffusion processes. In the case of submicrometer grain sizes, where intragranular deformation can be interpreted as being dominant at moderate temperatures, the strength of the material can be increased by grain refinement, either by grain-size hardening or the Hall-Petch effect. This tendency can be understood by considering the grain boundary as a barrier to the movement of dislocations. However, grain-size softening has been reported in cases where the grain size is of nanometer dimensions. ${ }^{1,2}$ Hence, when we consider the influence of the grain size on the strength of crystalline materials (i.e., the transition from grain-size hardening to grain-size softening), we can postulate that there should be an optimum grain size that would provide an optimized strength for all such materials. ${ }^{3}$ In recent times, optimum grain sizes were confirmed to be $10-40 \mathrm{~nm}$ via experiments. ${ }^{4-6}$ These grain sizes correspond well to the critical grain size for face-centeredcubic (fcc) metals to activate a dislocation source in a grain. ${ }^{7}$ In addition, a nonresidual dislocation network in nanograins during deformation has been confirmed. Therefore, the internal grain structure implies the existence of characteristic deformation mechanisms that do not involve dislocations. ${ }^{8}$ In other words, an understanding of the conditions for optimum grain size for polycrystalline materials, i.e., an understanding of the dependence of deformation mechanisms on grain size, is probably a key aspect for future material design.

Atomic simulation is a powerful tool for studying nanocrystalline materials because it can directly express the movement of dislocations and the structure of grain boundaries at the atomic level within limitations of treatable time and space scale; hence, it should be noted that a dislocation climb in a crystal structure is hard to express in molecular dynamics simulation due to time-scale limitations. Several meaningful studies have investigated nanocrystalline metallic materials by atomic simulation, e.g., the inverse HallPetch relation, ${ }^{9,10}$ the relationship between grain boundary structures and deformation mechanisms, ${ }^{11-15}$ and the relationship between intragranular deformation and internal structures of nanograins. ${ }^{16-23}$ Additionally, optimum grain sizes for nanocrystalline $\mathrm{Al}$ and $\mathrm{Cu}$ have been reported by Yamakov et al..$^{24}$ and Schiøtz et al. ${ }^{25}$ These values are approximately $18 \mathrm{~nm}$ for $\mathrm{Al}$ and $10-15 \mathrm{~nm}$ for $\mathrm{Cu}$. Yamakov et $a l .{ }^{26}$ also proposed an extremely interesting deformation mechanism map for nanocrystalline metals based on the idea that the optimum grain size can be controlled by the stacking fault energy, the elastic properties of the metals, and the applied stress level. They predicted that the optimum grain size increases with decreasing stacking fault energy because stacking faults forming in nanograins by a partial dislocation motion prevent further crystal slip. Thus, stacking faults in nanograins can influence the dominant deformation mechanisms: intergranular or intragranular deformation. However, Frøseth et al. ${ }^{27}$ showed that even in the case of nanocrystalline materials with stacking faults, intragranular deformation 


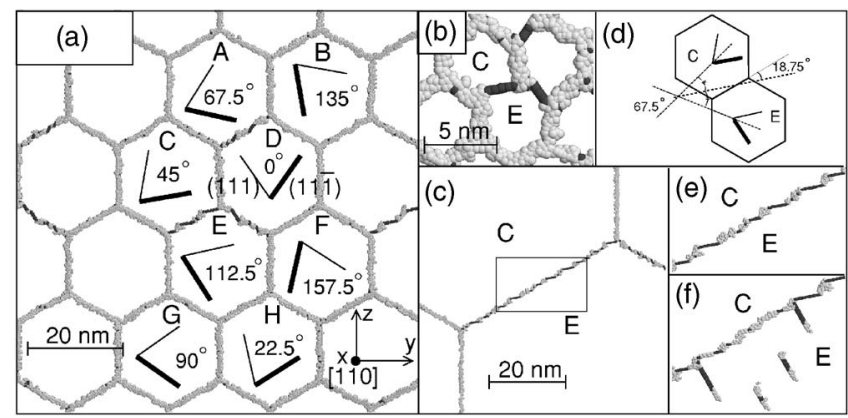

FIG. 1. Analytical models and grain boundary structures of nanocrystalline materials with different grain sizes. The light gray and the dark gray atoms show the defect and the local hep structure, respectively. (a) $20 \mathrm{~nm}$, (b) $5 \mathrm{~nm}$, and (c) $80 \mathrm{~nm}$. Crystal orientations are shown in (a), where the thin and thick lines represent the (111) and $(11 \overline{1})$ atomic planes and the angle between the two planes represents the rotational angle around the [110] direction. (d) The geometrical relationship between the grain-C and grain-E. (e), (f) Grain boundary structure in the rectangular region in (c) at $\varepsilon$ $=0.00$ and $\varepsilon=0.03$. The grain boundary structure is $\Sigma 3$ with steps; the steps have the potential to become dislocation sources.

occurs by twin boundary migration and deformation twinning. Consequently, the factors that are most influential in determining the optimum grain size remain unknown. In this study, we examine the dependence of the relationship between intergranular and intragranular deformation on grain size by simulating the tensile deformation of nanocrystalline Al with a regular hexagonal grain shape. Finally, the optimum grain sizes obtained using the methods given in this paper and in previous studies are compared.

\section{METHODOLOGY}

The model for nanocrystalline $\mathrm{Al}$ uses the embedded atom method of Mishin et al. ${ }^{28}$ This method accurately reproduces the energy values of stacking faults. We consider 13 models with grain sizes $d$ ranging from $5 \mathrm{~nm}$ to $80 \mathrm{~nm}$. The analysis models comprise unit structures that consist of eight hexagonal grains with crystal orientation in the $x$ direction that is fixed to $\langle 110\rangle$, which is the same direction used in the previous quasi-two-dimensional models ${ }^{17}$ [refer to Fig. 1(a)]. The size of the unit structure is determined uniquely by grain size $d$. Hence, the number of unit structures in the $y$ and $z$ direction is determined to make the size of the entire analysis model as similar as possible, e.g., $12 \times 12(y \times z)$ for a $5 \mathrm{~nm}$ model and $1 \times 1$ for a $60 \mathrm{~nm}$ model. There exists $1.2-3.2$ million atoms in each model. A periodic boundary condition is adopted in all directions. A tensile deformation in the $z$ direction is performed at a strain rate of $8 \times 10^{8} 1 / \mathrm{s}$, whereas deformation in the $y$ direction is carried out by maintaining the stress $\sigma_{y y}$ as zero by the Parrinello-Rahman method. ${ }^{29}$ The length of all models in the $x$ direction is approximately $1.1 \mathrm{~nm}$ and there are eight atomic planes. The temperature is maintained at $300 \mathrm{~K}$ during all simulations. The local face-centered-cubic (fcc), hexagonal-close-packed (hcp) crystal structure and defect atomic structures are classified using common neighbor analysis (CNA). ${ }^{30}$

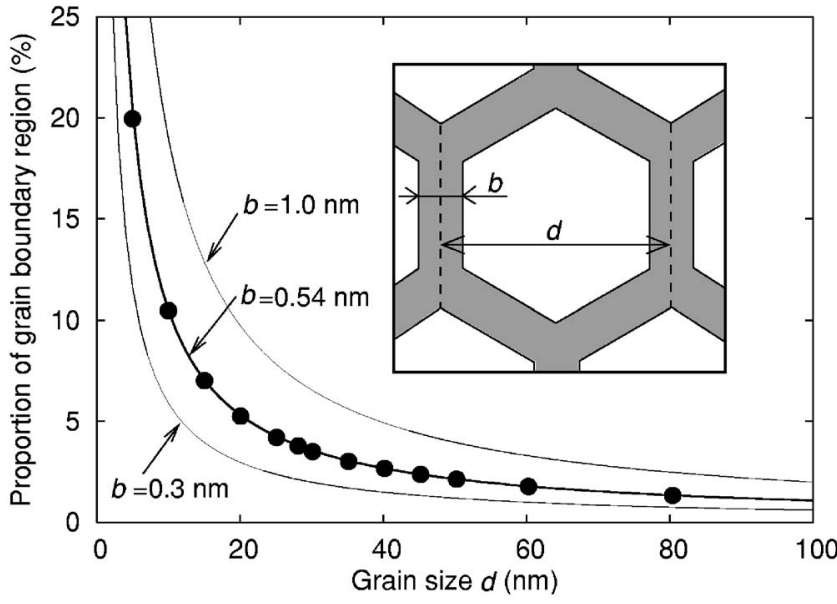

FIG. 2. Relative proportion of the grain boundary region in simulated nanocrystalline metals $f_{\mathrm{gb}}^{\mathrm{MD}} ; \bullet$, vs grain size $d$. The solid curves represent $f_{\mathrm{gb}}^{\text {ideal }}$ estimated by an ideal model with a grain boundary thickness $b$ shown in the inset. The average grain boundary thickness of each atomic model can be estimated to be constant at $5.4 \AA$; therefore, it does not depend on grain size $d$.

Due to the geometric restrictions on the simulation cell, 60-degree dislocations on two slip planes, such as $(11 \overline{1})$ and (111) in Fig. 1(a), fundamentally dominate intragranular deformation. The distance between two partial dislocations of a perfect dislocation is influenced by the crystal orientation because the resolved shear stresses applied to the leadingand trailing-partial dislocations are not identical. ${ }^{17}$

\section{RESULTS}

\section{A. Nanocrystalline structures}

Figures $1(a)-1(c)$ show grain boundary structures in a relaxed state for $20 \mathrm{~nm}, 5 \mathrm{~nm}$, and $80 \mathrm{~nm}$, respectively. First, we examine the dependence of the grain boundary thickness $b$ on grain size. In an ideal two-dimensional nanocrystalline with a regular hexagonal grain shape, the theoretical proportion of grain boundary region $f_{\mathrm{gb}}^{\text {ideal }}$ is estimated as $1-(d$ $-b)^{2} / d^{2}$, where $b$ and $d$ are the grain boundary thickness and grain size, respectively. The relationship is established based on the assumption that $b$ is constant. On the other hand, the computational proportion of the grain boundary region $f_{\mathrm{gb}}^{\mathrm{MD}}$ is defined as $1-N^{\mathrm{fcc}} / N$, where $N^{\mathrm{fcc}}$ and $N$ are the number of atoms in a local fcc structure classified using CNA and the number of total atoms in each model, respectively. The solid curves in Fig. 2 represent $f_{\mathrm{gb}}^{\text {ideal }}$ with a grain boundary thickness $b=0.30,0.54$, and $1.00 \mathrm{~nm}$, respectively; the solid circles represent $f_{\mathrm{gb}}^{\mathrm{MD}}$ in our atomic models. The plots of $f_{\mathrm{gb}}^{\mathrm{MD}}$ lie on the curve of $f_{\mathrm{gb}}^{\text {ideal }}$ with $b=0.54 \mathrm{~nm}$. This proves that the average grain boundary thickness $b$ in our atomic models does not depend on grain size $d$, as reported previously, ${ }^{31}$ and the grain boundary thickness $b$ cannot be neglected when the grain size $d$ decreases. In the case of a $10 \mathrm{~nm}$ grain size, $f_{\mathrm{gb}}^{\mathrm{MD}}$ is approximately $10.5 \%$. Therefore, it consists of two distinct regions, i.e., well-ordered and disordered atomic regions, even in the case of single-component nanocrystalline materials. 


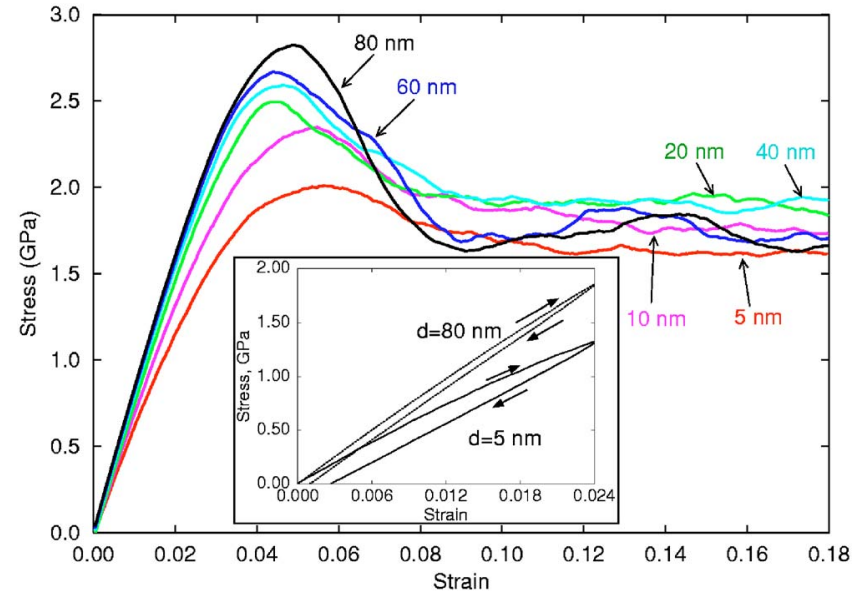

FIG. 3. (Color online) Stress-strain curves under tensile loading at $300 \mathrm{~K}$. The inset shows test results for loading and unloading when $d=5$ and $80 \mathrm{~nm}$. There is a permanent strain of 0.0005 for a grain size of $80 \mathrm{~nm}$ and 0.0027 for a grain size of $5 \mathrm{~nm}$ that is not caused by dislocation events after loading and unloading tests.

Secondly, we examine the dependence of grain boundary structures on grain size. We can confirm that the regular hexagonal grain shape in the initial configuration changes after relaxation for the $5 \mathrm{~nm}$ model and that the local grain boundary structure differs from that of other models. For example, as shown in Fig. 1(d), the grain boundary misorientation angle between the grain-C and grain-E is almost that of the $\Sigma 3\langle 110\rangle$ tilt grain boundary (70.5 degrees); however, the difference of the inclination angle of the grain boundary is approximately 19 degrees from the crystallographically symmetric interface between the grain- $\mathrm{C}$ and grain- $\mathrm{E}$, shown by the broken line in Fig. 1(d). That structure can be regarded as the $\Sigma 3\langle 110\rangle$ tilt grain boundary. After relaxation, the grain boundary between grain-C and grain-E in $5 \mathrm{~nm}$ models migrates and consists of only hcp atoms (dark-colored atoms), i.e., only the $\Sigma 3\langle 110\rangle$ tilt grain boundary. On the other hand, when $d=20$ or $80 \mathrm{~nm}$, as in Figs. 1(a) and 1(c), the inclination of the whole grain boundary plane between grain- $\mathrm{C}$ and grain-E does not change after relaxation and the interfaces consist of the $\Sigma 3\langle 110\rangle$ tilt grain boundary with steps which accommodate the geometrical restriction against the formation of the $\Sigma 3\langle 110\rangle$ tilt grain boundary as shown in Fig. 1(e), which is an enlarged picture of the square region in Fig. 1(c). Figure 1(f) shows the atomic configuration when $\varepsilon=0.03$ in the same region shown in Fig. 1(e) and reveals that these steps become dislocation sources. Consequently, the local grain boundary structure depends on grain size $d$ because of the difference in the distance between triple junctions.

\section{B. Mechanical properties}

Figure 3 shows stress-strain curves under tensile loading. A decrease in the slopes of the stress-strain curves as grain size decreases can be confirmed. This tendency corresponds well to the dependence of the $f_{\mathrm{gb}}^{\mathrm{MD}}$ on grain size in Fig. 2. To understand the cause for the decrease in the slope of the stress-strain curve, unloading tests are performed. A perma-

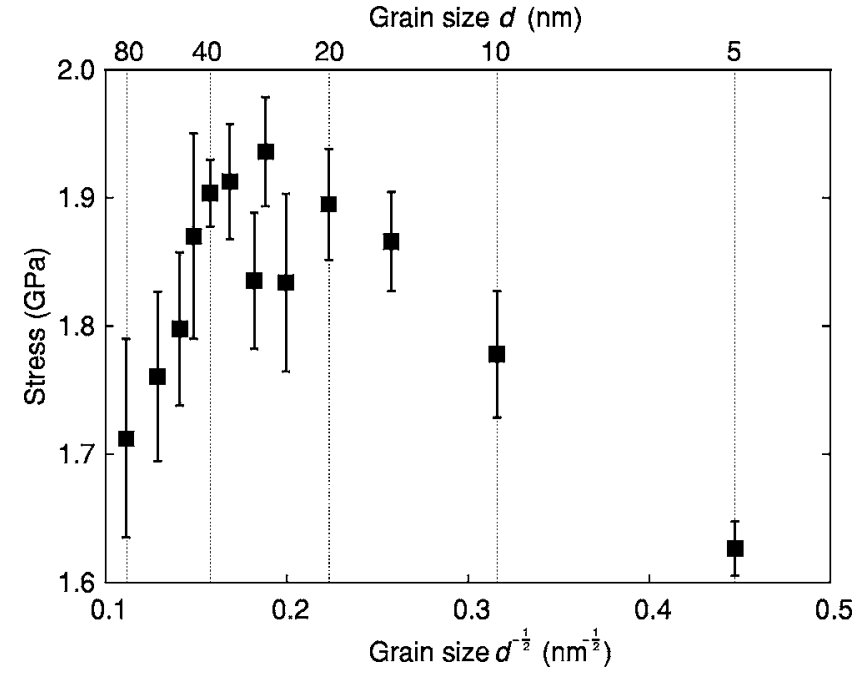

FIG. 4. Flow stress $\sigma_{\mathrm{f}}$; vs $d^{-1 / 2}$. The flow stress is estimated by averaging the tensile stress after a strain $\varepsilon=0.1$. A transition from the Hall-Petch relation to the inverse Hall-Petch relation can be observed. The maximum strength appears in $d \approx 30 \mathrm{~nm}$.

nent strain remains for each model, i.e., 0.0005 for $80 \mathrm{~nm}$ and 0.0027 for $5 \mathrm{~nm}$, when unloading is performed on the 5 $\mathrm{nm}$ and $80 \mathrm{~nm}$ models at $\varepsilon=0.024$ (as shown in the inset in Fig. 3). No dislocation processes occur until $\varepsilon=0.024$. Therefore, intergranular deformation should occur before the intragranular deformation becomes active. In other words, the decrease in the slopes of the stress-strain curves prior to the peak stress could have occurred by the release of stored elastic energy by local plastic deformation in grain boundary regions. Consequently, the resistance to intergranular deformation becomes smaller than the resistance to intragranular deformation as grain size decreases. Values of the strain at the peak stresses for $5 \mathrm{~nm}$ and $10 \mathrm{~nm}$ models become larger than those for other models. This implies that the yield mechanism changes around that specific grain size, i.e., a transition from an intragranular to an intergranular yield mechanism occurs around that size.

In these simulations, nanocrystalline models deform under the following severe conditions: tensile deformation speed is very high, none of the grains contain any defect in the initial state, and crystal slips have geometrical restrictions, increasing the stress to values higher than those in the experiments; further, the flow stress becomes oscillating as the initial grain size becomes large as shown in Fig. 3, because the total number of grains in models with a large grain size is less. However, the dependence of the grain size $d$ on the flow stress $\sigma_{\mathrm{f}}$ beyond the peak stress values shows a transition from grain-size hardening to grain-size softening. Figure 4 shows the relationship between the flow stress $\sigma_{\mathrm{f}}$ and the inverse square root of grain size $d^{-1 / 2}$. The flow stress is estimated by averaging the tensile stress after a strain $\varepsilon=0.1$. The figure shows that the flow stress increases as the grain size decreases to $30 \mathrm{~nm}$, which illustrates the Hall-Petch effect. On the other hand, grain-size softening can be observed as the grain size becomes smaller than $30 \mathrm{~nm}$, which illustrates the inverse Hall-Petch effect. Consequently, in this simulation, the maximum strength for nanocrystalline $\mathrm{Al}$ appears in the region around $d \approx 30 \mathrm{~nm}$. 


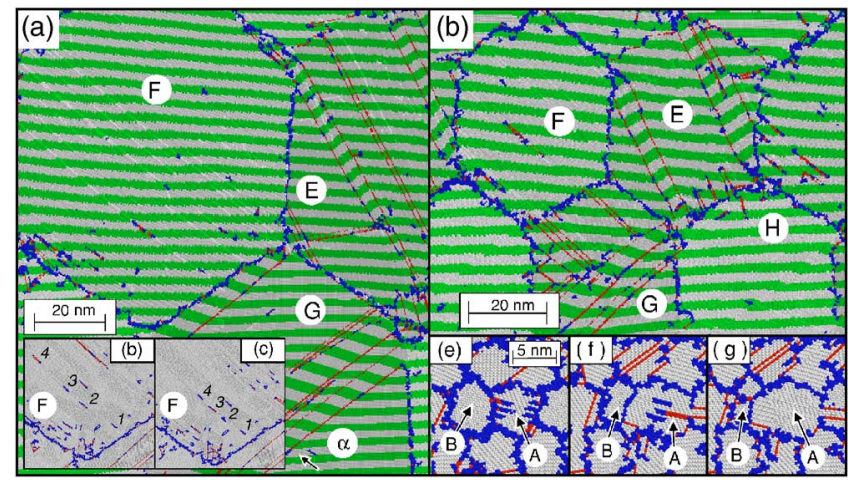

FIG. 5. (Color online) Intragranular deformation in nanocrystalline metals. The internal structure of nanocrystalline $\mathrm{Al}$ when a strain $\varepsilon=0.12$. The darkest gray and the dark gray atoms represent the local defect and the hcp structure, respectively, whereas the medium gray and light gray atoms represent the local fcc structure. The medium gray and light gray stripes are marked on the initial atomic configurations to identify intragranular and intergranular deformations. (a) $d=80 \mathrm{~nm}$ in the grain-size hardening region. Some dislocations are simultaneously active in the grains. (b), (c) a pile-up of dislocations $1-4$ in grain-F in the vicinity of the grain boundary for $d=80 \mathrm{~nm}$ when $\varepsilon=0.164$ and 0.176 . (d) $d=40 \mathrm{~nm}$ around the optimum strength region. The grains still deform homogeneously, so no remarkable intergranular deformation occurs. (e), (f), and (g) $d=5 \mathrm{~nm}$ in the grain-size softening region. Grain boundary migration occurs when grain boundary dislocations among the grain-A and grain-B are active and penetrate grain-A, where $\varepsilon$ $=0.060,0.112$, and 0.120 , respectively. Nanocrystals in the grainsize softening region cannot maintain dislocation cores within themselves.

\section{Intragranular deformation in nanocrystalline metals}

We then investigate intragranular deformation that is closely related to grain-size hardening. Figure 5 shows the internal structure of the nanocrystalline $\mathrm{Al}$ in the form of atomic models. Figure 5(a) shows atomic configurations of $80 \mathrm{~nm}$ grains at a strain 0.12 . Several dislocations can move simultaneously in grain-F. Figures 5(b) and 5(c) also show atomic configurations in grain-F at a strain 0.164 and 0.176 , respectively. A pile-up of dislocations 1-4 toward the grain boundary among grain-F and grain- $G$ can be observed in grain-F, but the respective crystal glide planes of dislocations 1-4 are not identical. A pile-up of dislocations facilitates activation of intragranular deformation in adjacent grains because the stress concentration that is attributable to a pile-up of dislocations near the grain boundaries occurs, illustrating the Hall-Petch mechanism.

Figure 5(d) shows atomic configurations of $40 \mathrm{~nm}$ grains at a strain 0.12 . The number of dislocations in grain-F becomes less than that of $80 \mathrm{~nm}$ grains. Therefore, a transition of intragranular deformation to the next grain becomes more difficult as grain size decreases, because a pile-up of dislocations cannot be formed in nanograins. Moreover, the mean free path of the dislocation glide decreases as the grain size decreases, namely there are several barriers to the dislocation movement. The grains in $40 \mathrm{~nm}$ and $80 \mathrm{~nm}$ models still deform homogeneously, as shown in Figs. 5(a)-5(d). Therefore, no remarkable intergranular deformation occurs. Con- sequently, grain-size hardening in Fig. 4 is caused by the grain boundary role as a barrier to intragranular deformation.

Next we examine the case of intragranular deformation in even smaller grains. Figures $5(\mathrm{e})-5(\mathrm{~g})$ show atomic configurations of $5 \mathrm{~nm}$ grains at strains of $0.060,0.112$, and 0.120 , respectively. As the strain increases, grain boundary dislocations among grain-A and grain-B become active and penetrate grain-A. However, the nanograin cannot maintain the elastic energy of dislocation cores within itself. Thereby, grain boundary migration occurs and the grain growth of grain-A can be confirmed. Accordingly, nanograins in this grain size region are presumed to be too small to deform by perfect dislocation since nanograins cannot maintain the stability of these defects.

Nanocrystalline $\mathrm{Al}$ deformation twins have been observed in experiments ${ }^{32,33}$ and atomic simulations ${ }^{17}$; they have also been observed in this simulation. It should be noticed that quasi-two-dimensional models are adopted in this and previous studies. ${ }^{17}$ Therefore, the distance between two partial dislocations of a 60-degree dislocation is different from that of a perfect edge or screw dislocation under an external force, because the resolved shear stresses on the two partials of the 60-degree dislocation are not equal. The twin boundary acts as a barrier to dislocations; hence, the appearance of a twin boundary can be interpreted as "grain refinement." For example, the dislocation $\alpha$ in Fig. 5(a), which is moving toward a twin boundary, cannot actually pass through that boundary. On the other hand, twin boundaries can migrate by crystal slips; thereby, nanograin Al can change the grain shape through deformation twinning. ${ }^{27}$ Consequently, the mechanical properties of nanocrystalline Al with deformation twinning might show a unique characteristic that differs from coarse-grained Al.

\section{Intergranular deformation in nanocrystalline metals}

The dominant intergranular deformation for polycrystalline metals under high temperature and low applied stress is known to be the grain boundary diffusion mechanism, Coble creep, where the relation $\dot{\varepsilon} \propto d^{-n}$ is observed and usually $n$ $=3$. Yamakov et $a l .{ }^{34}$ showed that the value of $n$ reaches 2 when the grain boundary thickness $b$ becomes comparable to the grain size $d$. On the other hand, superplasticity by grain boundary sliding has been confirmed in nanocrystalline metals under a high strain rate and moderate temperature. ${ }^{35}$ In other words, intergranular deformation mechanisms change with changes in analysis conditions. In this section, we investigate intergranular deformation of nanocrystalline metals under a high strain rate and moderate temperature.

Figure 6(a) shows the atomic configuration at a strain 0.12 for $5 \mathrm{~nm}$ grains. The grain shapes change from the initial hexagonal shape. This tendency is different from the cases of the $40 \mathrm{~nm}$ and $80 \mathrm{~nm}$ models, as shown in Figs. 5(a)-5(d). Mismatches in the striped patterns having medium gray and light gray color at the grain boundaries between grain- $\mathrm{C}$ and grain-D can be observed. Therefore, grains shift their positions relatively by local grain boundary sliding. Figure 6(c) shows the equivalent strain at each atomic position between strains 0.00 and 0.12 . The dark color corresponds to the 


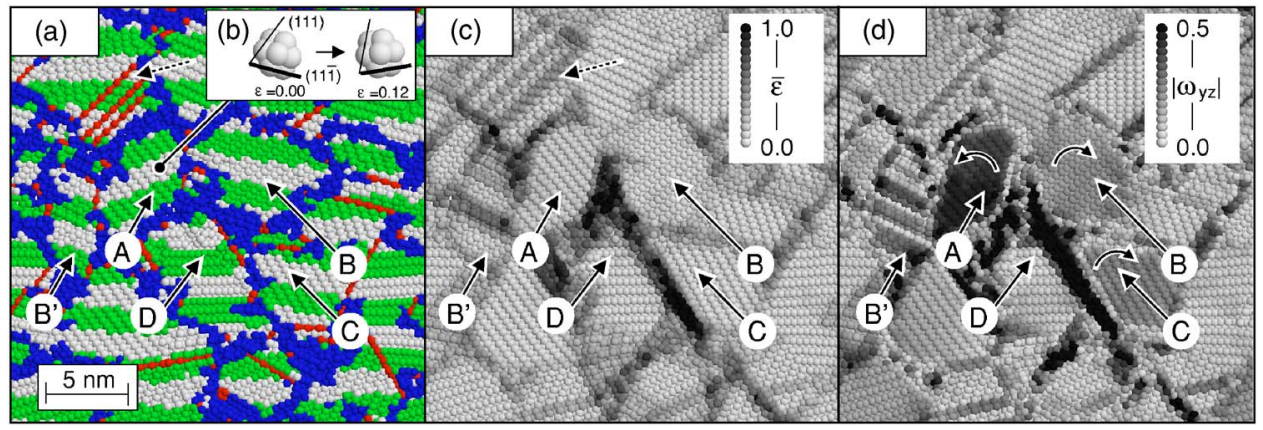

FIG. 6. (Color online) Intergranular deformation of nanocrystalline Al in $5 \mathrm{~nm}$ grain when strain $\varepsilon=0.12$. (a) Atomic configurations colored by the same rule as that in Fig. 5. No dislocation cores exist in the grains. Gaps in the stripes at the grain boundary between grain-C and grain-D are visible. (b) The crystal orientation of grain-A when $\varepsilon=0.00$ and 0.12 . (c) Distribution of the equivalent strain $\bar{\varepsilon}$ from a strain 0.00 and 0.12 . Dark colored atoms are observed at the grain boundary between grain-C and grain-D. Hence, grain boundary sliding occurs at this grain boundary. No dark colored atom exists in grain-A, grain-B, or grain-C. Therefore, no crystal slip occurs in these grains. (d) Distribution of the absolute value of an antisymmetric component of displacement gradient $\left|\omega_{y z}\right|$ from $\varepsilon=0.00$ and 0.12 . Grain-A, grain-B, and grain- $\mathrm{C}$ consist of dark colored atoms. Therefore, crystal rotation occurs without crystal slips in these grains. Arrows show the directions of the respective crystals' rotations.

larger value; for example, the atoms in a crystal slip plane shown in Fig. 6(a) by the dashed arrow are shown in Fig. 6(c) by the dark, dashed arrow. Several dark-colored atoms exist in the grain boundary region between grain- $\mathrm{C}$ and grain-D. Hence, grain boundary sliding occurs in the grain boundary.

In general, grain boundary sliding produces geometrical misfits at triple junctions such as cracks, which is one of the causes of intergranular fracture. Therefore, accommodation mechanisms for misfits are required to obtain large elongation. One such mechanism would be a grain boundary diffusion creep mechanism ${ }^{24}$ and another possibility is a grain rotation mechanism proposed by theoretical analyses ${ }^{36,37}$ and observed in this simulation as shown below. Theoretical analyses show that a gliding grain boundary dislocation causing grain boundary sliding splits into climbing grain boundary dislocations at the triple junction, and then the grain rotation occurs through the motion of climbing grain boundary dislocations which compose a grain boundary disclination from a large-scale viewpoint, and this process is an energetically practical deformation mechanism in nanocrystalline materials (refer to Fig. 7). ${ }^{36}$ Further, disclination dipoles are experimentally observed in body-centered-cubic iron that had undergone severe plastic deformation. ${ }^{38}$ On the other hand, in our simulation, Fig. 6(d) shows the absolute value of an antisymmetric component of displacement gradient $\left|\omega_{y z}\right|$ between strains 0.00 and 0.12 at each atomic position in the same region in Fig. 6(a). Grain-A, grain-B, and grain-C consist of dark colored atoms; hence, crystal grain rotation occurs without crystal slips because there exist no dark-colored atoms with a large equivalent strain in these grains in Fig. 6(c). Figure 6(b) shows the crystal orientation for grain-A when the strain $\varepsilon=0.00$ and 0.12 , and the rotational angle of grain-A is approximately 25 degrees. Consequently, the grain rotation mechanism can accommodate the geometrical misfit caused by grain boundary sliding between grain- $\mathrm{C}$ and grain-D. Moreover, the striped patterns for grain-A are rotated in a counterclockwise direction, while those for grain-B and grain- $\mathrm{B}^{\prime}$ are rotated in a clockwise direction. Thus, no mismatch in the striped patterns at the grain boundary between grain- $\mathrm{A}$ and grain-B and between grain-A and grain-B' can be observed. These results support the proporsed theory ${ }^{36}$ of rotation mechanism with respect to a dipole of wedge disclinations, which occur on the grain boundaries, as shown in Fig. 7(b), and we can presume that dislocations can climb more easily in a disordered region than in a well-ordered region. In comparison to the grain boundary diffusion mechanism, the grain rotational accommodation mechanism confirmed in our atomic simulations can take advantage of the accommodation speed. Therefore, this mechanism is effective for superplastic deformation under a high strain rate and moderate temperature.

Next, we address cooperative intergranular deformation of nanocrystals observed in the $5 \mathrm{~nm}$ model. The resistance to grain boundary sliding in this study can be assumed to decrease as the grain size decreases because the initial zigzag

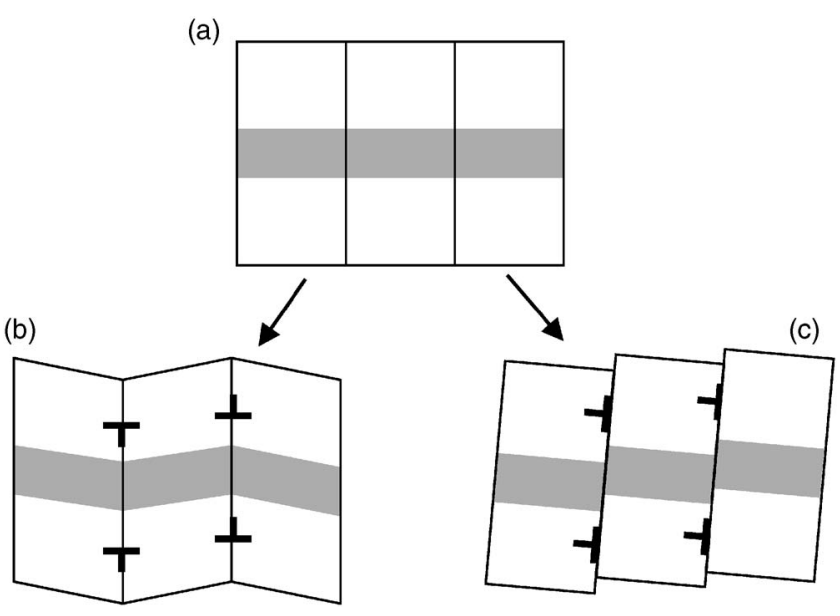

FIG. 7. Schematic figure showing grain rotation mechanisms. (a) Three grains marked with a gray line on each center in an initial state. Grain rotation occurring through climbing of grain boundary dislocations, i.e., grain boundary disclination dipoles (b) and gliding of grain boundary dislocations (c). These mechanisms make no misfit and misfit of gray lines at each grain boundary, respectively. 

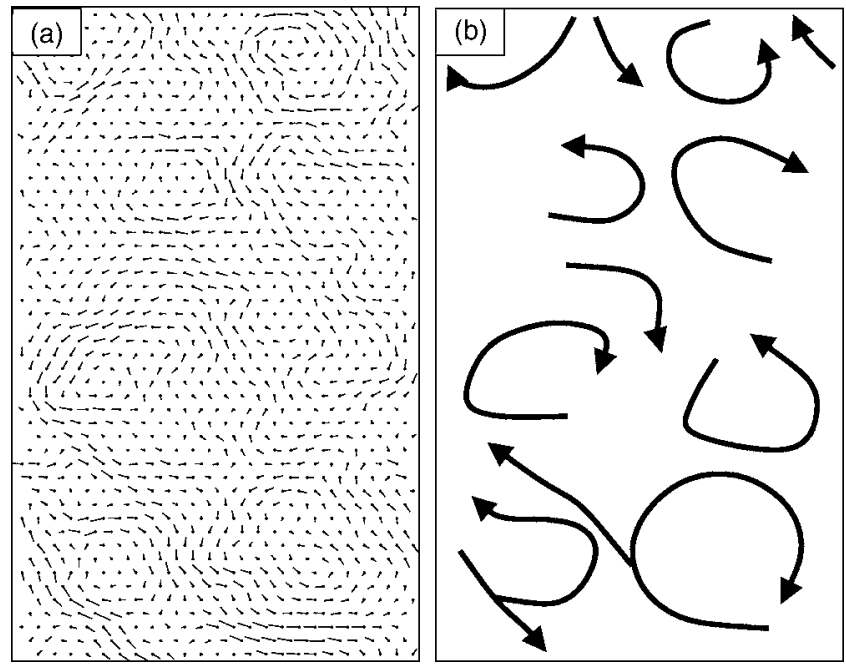

FIG. 8. (a) Different centers of gravity of each grain that are attributable to homogeneous deformation at $\varepsilon=0.12$. The solid circles are the centers of gravity of respective grains in the homogeneous deformation at $\varepsilon=0.12$. The line length corresponds to four times the value of the deviation between the center of gravity in the atomic simulation and in the homogeneous deformation. (b) Directions of the (mesoscale) cooperative deformation.

grain boundary arrangement can be changed easily to a wavy grain boundary arrangement by small atomic scale grain boundary migration. Figure 8(a) shows deviations of the center of gravity of each grain from the homogeneous deformation in the $5 \mathrm{~nm}$ model at $\varepsilon=0.12$. The solid circles correspond to positions of the center of gravity of each grain in the homogeneous deformed model. The line length shows the values of deviation. It should be noted that the line length is four times greater than the actual value of deviation. Several deviations appear in the $5 \mathrm{~nm}$ model. Hence, the grain boundary diffusion mechanism or Coble creep, whereby every crystal grain must deform homogeneously, is not the dominant deformation mechanism in this model. Figure 8(b) shows a conceptual image for the cooperative migration of nanograins shown in Fig. 8(a). This picture reveals mesoscale cooperative grain boundary sliding ${ }^{39}$ by the microscale mechanisms of grain boundary sliding, migration, and rotation, as shown in Fig. 6. Moreover, the mesoscale vortex size may strongly depend on macroscale boundary conditions. Therefore, this mesoscale deformation mechanism, i.e., the cooperative grain boundary sliding, governs the macroscopic mechanical properties and deformation mechanism. ${ }^{40}$

Consequently, under a high strain rate and moderate temperature, the cooperative grain boundary sliding consisting of local grain boundary sliding, migration, and rotation is the dominant intergranular deformation mechanism in the nanograin size region where grain-size softening occurs. In other words, the degree of difficulty of accommodation to geometrical misfits by grain boundary sliding is related to the resistance to intergranular deformation; hence, nanocrystalline metals deform primarily via intergranular deformation as grain size decreases.

\section{E. Optimum grain size in nanocrystalline metals}

The results obtained in this study suggest that the optimum grain size is controlled by the relationship between

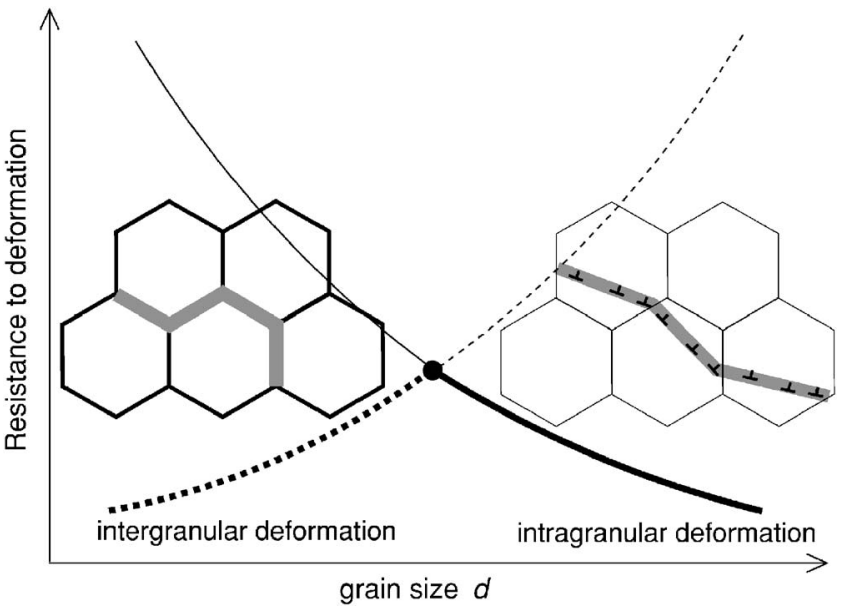

FIG. 9. Schematic figure showing the relationship between grain size $d$ and resistance to intragranular and intergranular deformation in nanocrystalline materials. It is convenient to consider that the resistance to intragranular deformation is proportional to $d^{-n}$ (the solid curve), e.g., $n=0.5$ for the Hall-Petch relation, and that the resistance to intergranular deformation is proportional to $d^{m}$ (the broken curve). Thereby, for the Coble creep, $m=3$ in coarse-grained polycrystalline materials and $m=2$ in nanocrystalline materials (Ref. 34).

resistance to intragranular deformation and intergranular deformation. Figure 9 shows a conceptual diagram of this relationship. The solid curve represents the resistance to intragranular deformation by dislocation movement. This resistance increases as the grain size decreases because the mean free path of the dislocation glide becomes small and the transition of intragranular deformation to the next grain by a pile-up of dislocations becomes difficult. Further, the broken curve represents resistance to intergranular deformation by grain boundary sliding, migration, and diffusion. This resistance decreases as the grain size decreases because the grain boundary thickness $b$ becomes equal to the grain size, and cooperative grain boundary sliding occurs easily under a high strain rate and moderate temperature. It is reasonable to infer that the region where the two curves of resistance to intergranular and intragranular deformation overlap, shown in Fig. 9, should correspond to the optimum grain size in nanocrystalline metals. In this simulation, the strongest material is observed when the grain size is approximately 30 $\mathrm{nm}$. This region corresponds to the region where the proportion of the grain boundary region increases dramatically, as shown in Fig. 2.

The difference between the optimum grain size of $\mathrm{Al}$ nanocrystalline materials obtained in this study and those obtained in a previous study by Yamakov et al. ${ }^{24}$ is of great interest. In both simulations, the analysis temperature is 300 $\mathrm{K}$; the strain rates show the same order: $\dot{\varepsilon} \simeq 10^{8} 1 / \mathrm{s}$, although the deformation conditions are different, i.e., constant stress for Yamakov's simulation and constant strain rate for our simulation. Yamakov's model ${ }^{24}$ used a different embedded atom method potential from ours. The stacking fault energy and lattice constant are $122 \mathrm{~mJ} / \mathrm{m}^{2}$ and $4.03 \AA$, respectively, in Yamakov's Al model while they are $158 \mathrm{~mJ} / \mathrm{m}^{2}$ and $4.05 \AA$, respectively, in our model. The splitting distance 
between two partial dislocations of perfect edge dislocations is $8.6 \AA$ for the former and $7.6 \AA$ for the latter. Hence, we can expect that approximately the same optimum grain size should be obtained in each simulation following the deformation mechanism map. ${ }^{26}$ However the optimum grain size obtained by our simulation is about twice as large as that obtained by Yamakov's simulation. Therefore, we presume that the difference in the optimum grain size is attributable to the difference in the resistance to the intragranular deformation. In Yamakov's simulation, the grain shape is the threedimensional rhombic dodecahedron shape which can express the full 12 crystal slip systems in fcc metals. However, in our simulation, the grain shape is the two-dimensional idealhexagonal prismatic shape which restricts crystal slips on only two slip planes. Thus, in the case of the Yamakov's model $^{24}$ the resistance to the intragranular deformation became less than that in our model. On the other hand, the resistance to intergranular deformation in both models can be inferred to be equivalent because each model comprises crystal grains in the regular grain shape and size, respectively. Consequently, the intersection point of the resistance curves in Yamakov's model, shown in Fig. 9, shifts to that with a smaller grain size than in our model, and the optimum grain size in Yamakov's study is smaller than that in this study. These results make clear that the optimum grain size is determined by the relationship between the resistance to intergranular and intragranular deformation.

Our Al model can easily generate stacking faults in grains because of the geometrical restriction to dislocation movement. In such a case, the simulated material in this study can be regarded as a hypothetical material with low stacking fault energy; in other words, the effective stacking fault energy of our Al model might be smaller than that of Yamakov's Al model. This relationship between the optimum grain size and effective stacking fault energy is in agreement with the prediction by the deformation-mechanism map proposed by Yamakov et al. ${ }^{26}$ as mentioned previously. It should be noted that there is a grain-size dependence of the threshold stress to nucleate a dislocation from a grain boundary as the role of dislocation sources in nanocrystalline materials, $\sigma$ $\propto 1 / d,{ }^{32}$ because the source size can be approximately estimated as the grain size $d$. This relation is expected to be one of the grain-size hardening mechanisms in nanocrystalline metals. ${ }^{26}$ However, this relation cannot be expressed in this simulation because the minimum thickness of the simulation cell in the $x$ direction in Fig. 1(a) is constant in all our simulations, i.e., there is no grain-size dependence of a dislocation source in our models. Nevertheless, the grain-size hardening can be confirmed even in our simulations. Thus, we can presume that the characteristic length to the grain-size hardening mechanism in nanocrystalline materials is not only the size of the dislocation source but also the size of the mean free path of the dislocation glide that is closely related with a pile-up of dislocation mechanisms as considered in coarse-grained polycrystalline metals. Moreover, intragranular deformation by a partial dislocation movement leaving a stacking fault in grains can occur even in larger grain size regions than the optimum grain size, such as the grain-size hardening region in our simulations (Fig. 5) and in the previous simulation for $\mathrm{Cu}^{25}$ Therefore, it is still unclear that stacking faults and twin boundaries in nanograins have a great influence on the optimum grain size of polycrystalline metals.

\section{CONCLUSIONS}

The dependence of intergranular deformation and the intragranular deformation on grain size, and the optimum grain size of aluminum nanocrystalline materials are studied using molecular-dynamics simulations. In these simulations, the optimum grain size for the strength of $\mathrm{Al}$ nanocrystalline materials is about $30 \mathrm{~nm}$. In grain-size hardening regions, nanocrystalline metals are deformed by crystal slips and pile-up dislocations are confirmed. In grain-size softening regions, cooperative grain boundary sliding is observed and geometrical misfits by grain boundary sliding are accommodated by the grain rotational mechanism. The difference in optimum grain sizes of $\mathrm{Al}$ nanocrystals in this study and in a previous study implies that the optimum grain size is determined by the relationship between the resistance to intergranular and intragranular deformation. The resistance to intergranular and intragranular deformation might be influenced by the analysis condition, e.g., strain rate or temperature. In other words, we can presume that the optimum grain size is not an inherent value of a material. From this perspective, we will continue to study the analysis condition dependence of optimum grain size.

\section{ACKNOWLEDGMENTS}

The authors gratefully acknowledge support from the Japan Society for the Promotion of Science (JSPS) (T.S.), from the Ministry of Education, Science, Sports and Culture, Grant-in-Aid for Young Scientists (B), 2004, 16760063 (T.S.), from New Energy and Industrial Technology Development Organization (NEDO) of Japan, and the Strategic Research Base, Handai Frontier Research Center, supported by the Japanese Government's Special Coordination Fund for Promoting Science and Technology (A.N. and H.K.).
${ }^{1}$ A. H. Chokshi, A. Rosen, J. Karch, and H. Gleiter, Scr. Metall. 23, 1679 (1989).

${ }^{2}$ G. E. Fougere, J. R. Weertman, R. W. Siegel, and S. Kim, Scr. Metall. Mater. 26, 1879 (1992).

${ }^{3}$ S. Yip, Nature (London) 391, 532 (1998).
${ }^{4}$ P. G. Sanders, J. A. Eastman, and J. R. Weertman, Acta Mater. 45, 4019 (1997)

${ }^{5}$ R. A. Masumura, P. M. Hazzledine, and C. S. Pande, Acta Mater. 46, 4527 (1998).

${ }^{6}$ A. S. Khan, H. Zhang, and L. Takacs, Int. J. Plast. 16, 1459 
(2000).

${ }^{7}$ M. Legros, B. R. Elliott, M. N. Rittner, J. R. Weertman, and K. J. Hemker, Philos. Mag. A 80, 1017 (2000).

${ }^{8}$ Z. Budrovic, H. Van Swygenhoven, P. M. Derlet, S. Van Petegem, and B. Schmitt, Science 304, 273 (2004).

${ }^{9}$ J. Schiøtz, T. Vegge, F. D. Di Tolla, and K. W. Jacobsen, Nature (London) 391, 561 (1998).

${ }^{10}$ T. Shimokawa, A. Nakatani, and H. Kitagawa, JSME Int. J., Ser. A 47, 83 (2004).

${ }^{11}$ H. Van Swygenhoven and P. M. Derlet, Phys. Rev. B 64, 224105 (2001).

${ }^{12}$ H. Van Swygenhoven, A. Caro, and D. Farkas, Mater. Sci. Eng., A 309-310, 440 (2001).

${ }^{13}$ A. Hasnaoui, H. Van Swygenhoven, and P. M. Derlet, Acta Mater. 50, 3927 (2002).

${ }^{14}$ H. Van Swygenhoven, P. M. Derlet, and A. Hasnaoui, Phys. Rev. B 66, 024101 (2002).

${ }^{15}$ A. Hasnaoui, P. M. Derlet, and H. Van Swygenhoven, Acta Mater. 52, 2251 (2004).

${ }^{16}$ J. Schiøtz, T. Vegge, F. D. Di Tolla, and K. W. Jacobsen, Phys. Rev. B 60, 11971 (1999).

${ }^{17}$ V. Yamakov, D. Wolf, M. Salazar, S. R. Phillpot, and H. Gleiter, Acta Mater. 49, 2713 (2001).

${ }^{18}$ V. Yamakov, D. Wolf, S. R. Phillpot, and H. Gleiter, Acta Mater. 50, 5005 (2002).

${ }^{19}$ V. Yamakov, D. Wolf, S. R. Phillpot, and H. Gleiter, Acta Mater. 51, 1971 (2003).

${ }^{20}$ H. Van Swygenhoven, M. Spaczer, and A. Caro, Acta Mater. 47, 3117 (1999).

${ }^{21}$ H. Van Swygenhoven, M. Spaczer, A. Caro, and D. Farkas, Phys. Rev. B 60, 22 (1999).

${ }^{22}$ J. Schiøtz and S. L. Frederiksen, in IUTAM Symposium on Mesoscopic Dynamics of Fracture Process and Materials Strength, edited by H. Kitagawa and Y. Shibutani, Solid Mechanics and its Applications Vol. 115 (Kluwer Academic Publishers, Dordrecht,
2004), pp. 355-363.

${ }^{23}$ A. Nakatani, T. Shimokawa, R. Matsumoto, and H. Kitagawa, in IUTAM Symposium on Mesoscopic Dynamics of Fracture Process and Materials Strength (Ref. 22), pp. 365-380.

${ }^{24}$ V. Yamakov, D. Wolf, S. R. Phillpot, A. K. Mukherjee, and H. Gleiter, Philos. Mag. Lett. 83, 385 (2003).

${ }^{25}$ J. Schiøtz and K. W. Jacobsen, Science 301, 1357 (2003).

${ }^{26}$ V. Yamakov, D. Wolf, S. R. Phillpot, A. K. Mukherjee, and H. Gleiter, Nat. Mater. 3, 43 (2004).

${ }^{27}$ A. Frøseth, H. Van Swygenhoven, and P. M. Derlet, Acta Mater. 52, 2251 (2004).

${ }^{28}$ Y. Mishin, D. Farkas, M. J. Mehl, and D. A. Papaconstantopoulos, Phys. Rev. B 59, 3393 (1999).

${ }^{29}$ M. Parrinello and A. Rahman, J. Appl. Phys. 52, 7182 (1981).

${ }^{30}$ H. Jónsson and H. C. Andersen, Phys. Rev. Lett. 60, 2295 (1988).

${ }^{31}$ H. Van Swygenhoven, Science 296, 66 (2002).

${ }^{32}$ M. Chen, E. Ma, K. J. Hemker, H. Sheng, Y. Wang, and X. Cheng, Science 300, 1275 (2003).

${ }^{33}$ X. Z. Liao, F. Zhou, E. J. Lavernia, D. W. He, and Y. T. Zhu, Appl. Phys. Lett. 83, 5062 (2003).

${ }^{34}$ V. Yamakov, D. Wolf, S. R. Phillpot, and H. Gleiter, Acta Mater. 50, 61 (2002).

${ }^{35}$ S. X. McFadden, R. S. Mishra, R. Z. Valiev, A. P. Zhilyaev, and A. K. Mukherjee, Nature (London) 398, 684 (1999).

${ }^{36}$ M. Y. Gutkin, I. A. Ovid'ko, and N. V. Skiba, Acta Mater. 51, 4059 (2003).

${ }^{37}$ I. A. Ovid'ko, Science 295, 2386 (2002).

${ }^{38}$ M. Murayama, J. M. Howe, H. Hidaka, and S. Takaki, Science 295, 2433 (2002).

${ }^{39}$ M. G. Zelin and A. K. Mukherjee, Philos. Mag. A 68, 1183 (1993).

${ }^{40}$ V. E. Panin, Physical Mesomechanics of Heterogeneous Media and Computer-aided Design of Materials (Cambridge International Science Publishing, Cambridge, 1998). 